\title{
Will Patient Generated Health Data Accurately be Incorporated into the Electronic Health Record if used to Diagnose or Treat Patients?
}

\author{
Skrocki M* \\ Saginaw Valley State University, USA
}

*Corresponding author: Marilyn Skrocki, Saginaw Valley State University, 5558 Spring Knoll Lane, USA, Tel: 9894508032; Email: mskrocki@svsu.edu

\section{Commentary}

Volume 3 Issue 3

Received Date: June 05, 2020

Published Date: June 18, 2020

DOI: $10.23880 /$ jqhe- 16000167

\section{Commentary}

Patient-generated health data (PGHD), is a term encompassing all forms of data that patients provide on their own. The custodian of the Electronic Medical Record is the person who has been designated responsible for the care, custody, and control of the health record for such persons or institutions that prepare and maintain records of healthcare. The official custodian or designee should be authorized to certify records and supervise all inspections and copying or duplication of records. Authentication is an attestation that something, such as a medical record, is genuine. The purpose of authentication is to show authorship and assign responsibility for an act, event, condition, opinion, or diagnosis. Every entry in the health record should be authenticated and traceable to the author of the entry. If Patient Generated Health Data is used to diagnose or treat patients, will it meet the authentication and traceable requirements for the medical record? Is Block chain the savior to meeting such requirements?

\section{Patient Generated Health Data}

Patient-generated health data (PGHD) [1], is a term encompassing all forms of data that patients provide on their own. The working definition of patient-generated health data is " health-related data-including health history, symptoms, biometric data, treatment history, lifestyle choices, and other information-created, recorded, gathered, or inferred by or from patients or their designees (i.e., care partners or those who assist them) to help address a health concern." [2]. The potential value of PGHD use includes: Improving patient health outcomes, enhancing the patient experience, alerting care teams for early intervention, and taking advantage of new reimbursement models [3].
Patient-generated health data falls into two broad groups: historical data and biometric data. Historical data is the type that clinicians are familiar with obtaining from patients: It includes the patient's medical history, allergies, medications, family history and lifestyle features. Biometric data are health data gathered by consumer medical devices, such as blood glucose meters and fitness trackers. Though clinicians are quite familiar with data entry from FDAapproved medical devices such as blood glucose meters, pacemakers and pulmonary function units, data from a myriad of consumer-driven health devices (Fitbit and others) will soon need to be included in the EHR.

Patients are knowledgeable about their health status and are actively involved in maintaining their wellbeing. Advances in consumer technologies such as smartphones and wearable fitness devices make it possible for individual consumers to have more control over their personal health records than ever before, opening the way for them to become more directly engaged in their health. From mobile pulse oximeters to Fitbits to continuous glucose monitors, wearable's are seemingly everywhere. The Apple Watch now features both an atrial fibrillation to detect algorithms and an electrocardiogram that helps identify heart abnormalities. In addition, the Apple Watch can use its Movement Disorder API to monitor symptoms in patients with Parkinson's disease. Virtual assistants, such as Amazon's Alexa and modified WiFi now provide the capability to continuously measure and report vital signs and other health markers. Ochsner Health System in New Orleans has launched a digital health initiative for expectant women that provides remote pregnancy care via wireless blood pressure cuffs and scales and at-home urine test that send real-time results to an obstetrician. 
According to the Pew Research Center, wearable and embedded health-tracking devices will be truly ubiquitous by 2025 . These technologies have the capability to provide patients and their healthcare teams with an array of insights that can augment traditional medical care in exciting and surprising ways.

Medicare, as part of the Medicare Access and CHIP Reauthorization Act (MACRA) [4] of 2015, requires the clinician, by 2020 , to fulfill a measure of patient engagement for optimal reimbursement (MACRA: Disrupting the US health care system at all levels: Deloitte US, 2018) [4]. To fulfill a measure of patient engagement the clinician must receive data from a non-clinical setting (e.g. a patient's home) into the certified EHR technology for at least one unique patient seen by the eligible clinician. According to The Patient Health Information Capture Criterion, §170.315(e) (3), a certified Electronic Health Record (EHR) product must "enable a user to 1.) Identify, record, and access information directly and electronically shared by a patient (or authorized representative) 2) Reference and link to patient health information documents." (What are the PGHD-related criteria in the health IT rules and programs? 2017) [5].

The Health Information Technology for Economic and Clinical Health (HITECH) Act, enacted as part of the American Recovery and Reinvestment Act of 2009, was signed into law on February 17, 2009, to promote the adoption and meaningful use of health information technology. A significant part of that legislation was intended for the ability of patients to interact with their medical information via the Internet. The lines between and EMR, a personal health record, and a patient portal are blurring. As technological advances continue to advance or morph the definition of the medical record, we are faced with the continued challenge of capturing the data in an electronic format along with maintaining the ability to provide data in a printed medical record format if requested.

\section{Maintenance of a Medical Record}

Healthcare providers, under national HIPAA laws as well as regulatory and state laws, must maintain a medical record for each patient with whom they have a professional relationship. The American Health Information Management defines the medical record as "a record of a patient's health and medical history and is defined as "any relevant record made by a health care practitioner at the time of, or subsequent to, a consultation and/or examination or the application of health management" [6]. Information in the medical record supports the ongoing care for the patient by the physician and other providers.

Many obstacles and challenges are present when considering incorporating PGHD into the legal electronic health record. Clinicians' and Researchers' Data-Related Concerns include the accuracy of the PGHD, Increased clinician workload due to large volume of PGHD, Integration of PGHD with the medical record and relevance to clinical management, responsibility for acting based on PGHD and privacy and security of PGHD [1]. Literature to date simply provides the disclosure "before implementing the use of PGHD, conduct a scan of relevant federal and state law, regulations, and guidelines that impact the project, as well as of relevant organizational policies" [1].

The custodian of the EMR is the person who has been designated responsible for the care, custody, and control of the health record for such persons or institutions that prepare and maintain records of healthcare. The official custodian or designee should be authorized to certify records and supervise all inspections and copying or duplication of records. The Health Information Management professional or designee is often considered the custodian of the health record and may be called to testify to the admissibility of the record. He or she may be asked to verify the timeliness and normal business practices used to develop and maintain the health record [7].

Authentication is an attestation that something, such as a medical record, is genuine. The purpose of authentication is to show authorship and assign responsibility for an act, event, condition, opinion, or diagnosis. Every entry in the health record should be authenticated and traceable to the author of the entry. The Rules of Evidence indicate that the author of the entry is the only one who has knowledge of the entry. The Federal Regulations/Interpretive Guidelines for Hospitals [8] (482.24(c)(1)(i)) require that there be a method for determining that the author did, in fact, authenticate the entry. This process should be defined in Health Information Management written policies and procedures and substantiate the authentication of an entry in a legal process [9-12]. Though the industry has made outstanding progress in adopting EHRs, the practice of data acquisition from patients remains a subject of discussion without resolution. Is Block chain a possible resolution?.

The definition of the legal medical record is very broad and capturing documentation and the ability to produce it to those requesting is required by law [13-16]. In addition to its clinical significance, the medical record is also a legal document that can serve as evidence of the care provided. There is no national database for medical records. It is our responsibility as healthcare providers, innovators, administrators, and all who provide such technology for medical purposes to look for new ideas to assure the patient's entire medical record is recorded in its entirety and maintained for the required minimum time. 
Block chain is used for many purposes but does it particularly fit the requirements of inclusion of PGHD into the EHR? IBM in its publication of "Block chain for Dummies" shares a query to use to see if Block chain is needed for your business purposes. It breaks the concepts into four areas: Consensus: Does agreement across the business network that each transaction is valid provide some benefit, Provenance: Is the maintenance of a complete audit trail important, Immutability: Is it important that the train of transactions is tamper-evident, Finality: Is there a need for an agreed "system of record" across the business network? I would argue Patient Generated Health Data should require systems utilizing the ingenuity of Block chain.

\section{References}

1. A practical Approach to the Use of Patient-Generated Health Data (PGHD), Office of the National Coordinator for Health Information Technology.

2. (2018) Patient-Generated Health Data.

3. ONC: Office of the National Coordinator for Health Information Technology.

4. Copeland B, Bill Thomas S, Sarah (2018) MACRA: Disrupting the US health care system at all levels. Deloitte US.
5. (2017) What are the PGHD-related criteria in the health IT rules and programs? HealthIT.gov

6. (2012) Overview. Electronic health records.

7. (2005) Guidelines for Defining the Health Record for Disclosure Purposes. Journal of AHIMA 76(8).

8. The Federal Regulations/Interpretive Guidelines for Hospitals (482.24).

9. (2010) Connected Health standards. Ministry of health.

10. Gupta M (2018) Blockchain for dummies. John Wiley \& Sons.

11. (2017) HIPAA for Professionals. HHS Office of the Secretary, Office for Civil Rights, \& OCR. 16).

12. Matterson M (2018) Ready Aim Fire.

13. (2015) Sanctions for Spoliation and Destruction of Evidence in Michigan.

14. (2019) The democratisation of healthcare-opportunities and challenges.

15. Tiase VL (2017) Navigating the patient-generated health data deluge. Nursing Management 48(12).

16. The HL7 Affiliate Closest to Tomorrow. 